\title{
Quality Enhancement of Biomedical Images using Super-Resolution Method based on Wavelets
}

\author{
Ankita Jain \\ Student \\ ITM University \\ Gurgaon
}

\author{
Rekha Vig \\ Associate Professor \\ ITM University \\ Gurgaon
}

\begin{abstract}
Image Super Resolution (SR) has become a very gripping and predominant area of research to overcome the issues of limited resolution. Super resolution is a kind of technique used for obtaining an image of very high resolution by combining different low resolution images of the similar scene. It not only increases the size of an image but also restores the degraded image. It can be used sometimes in biomedical imaging to help doctors for making the correct diagnosis, in forensic investigations to extract even the minute information and in many other applications. This paper introduces a new approach based on wavelets where the interpolated high frequency sub band images obtained by discrete wavelet transform are incremented to the high frequency sub bands obtained by stationary wavelet transform. An intermediate stage has been introduced for estimating the high frequency sub band images by exploiting the difference image obtained by deducting the interpolated low frequency sub band image from the input image. Considerable improvement in the value of PSNR and MSE has been observed for biomedical images.
\end{abstract}

\section{Keywords}

Super Resolution, discrete wavelet transform, interpolation, stationary wavelet transform.

\section{INTRODUCTION}

The main purpose of Super Resolution [1] is to extract even the minute details from a given low resolution image which can prove very useful in some applications like in video surveillance, satellite imaging, biomedical imaging etc. Super Resolution can be categorized into two different types: single frame SR and multi frame SR [2]. Multi frame technique uses multiple numbers of low resolution images of the similar scene to produce a single high resolution image. On the other hand, single frame technique makes use of only a single input image for obtaining a good quality output image [3]. The image pixels are not enlarged in size rather they are increased in numbers.

So the basic idea is to augment the amount of pixels per unit area in an image. This can be achieved through various hardware based approaches-

(1) decreasing the size of the pixels

(2) increased chip size

With decrease in individual pixel size, pixel density increases but the image gets degraded because of the introduction of shot. noise. Therefore this is not the correct approach of obtaining high resolution image. Another approach mentioned above will add an extra cost to that device. Hence this method is also discarded. To overcome these hardware limitations, some signal processing SR algorithms [4] have been implemented as this approach is not just cost effective but it also utilizes the existing low resolution imaging systems.

Some of the existing super resolution algorithms include interpolation method [5], reconstruction based SR [6] and example learning based super resolution technique [7]. Li et al. used edge directed interpolation that provided good results as compared to normal interpolation technique. Reconstruction technique worked further better. Another super resolution technique proposed by Anbarzafari et al. is based on wavelet transform for obtaining a high quality output image.

In this paper, a new super resolution technique which is based on discrete wavelet transform and stationary wavelet transform has been proposed. The high frequency components of the image obtained by DWT [8] are interpolated and added to the high frequency components obtained by SWT [9-11]. An intermediate stage has been introduced for estimating the high frequency components (LH, HL and $\mathrm{HH}$ ) by using the difference image obtained by deducting the low frequency sub-band image (LL) from the input image in order to get a sharper image. Then inverse DWT is applied on high frequency components and the input image for obtaining a high resolution image as output.

\section{WAVELET TRANSFORM}

A wavelet is a mathematical tool that can be used in image processing, signal processing and data compression to facilitate multi resolution analysis.

For this Fourier transform could be used but the main problem is that it gives either the frequency information or time information at any given time. It does not provide both the information simultaneously.

For an image of size $\mathrm{n} \times \mathrm{n}$, the discrete Fourier transform can be given by:

$$
F(k, l)=\sum_{i=0}^{n-1} \sum_{j=0}^{n-1} f(i, j) e^{-i 2 \pi\left(\frac{k i}{n}+\frac{l j}{n}\right)}
$$

where $f(i, j)$ represents the image in spatial domain and $\mathrm{F}(\mathrm{k}, \mathrm{l})$ represents the Fourier image. The exponential part represents the basis function.

The inverse Fourier transform (IDFT) can be used to transform the Fourier image back to spatial domain as given below:

$$
f(i, j)=\frac{1}{n^{2}} \sum_{k=0}^{n-1} \sum_{l=0}^{n-1} F(k, l) e^{i 2 \pi\left(\frac{k i}{n}+\frac{l j}{n}\right)}
$$


where $\mathrm{n}^{2}$ is the normalisation term used here. A key advantage of using wavelet transform over Fourier transform is that it provides both the frequency and location information simultaneously.

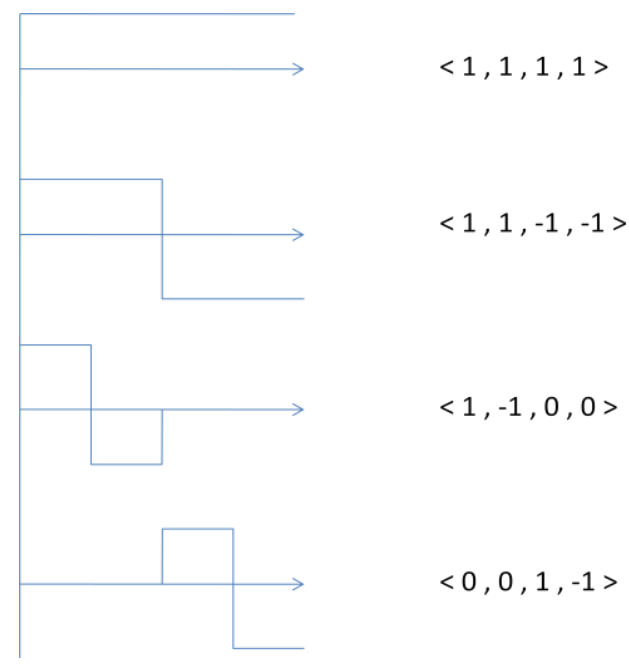

Figure 1: Haar Wavelet

\subsection{Discrete wavelet transform}

DWT is one of the most common wavelet transform that is being used these days in image processing. In this work, Haar wavelet is being used which is one of the most common square shaped function that all together form a wavelet basis as shown in figure 1 .
The Haar's mother wavelet function is given by :

$$
\psi(t)=\left\{\begin{array}{cc}
1 & 0 \leq t<\frac{1}{2} \\
-1 & \frac{1}{2} \leq t<1 \\
0 & \text { otherwise }
\end{array}\right\}
$$

and its scaling function is given by :

$$
\varphi(t)=\left\{\begin{array}{ll}
1, & 0 \leq t \leq 1 \\
0, & \text { otherwise }
\end{array}\right\}
$$

Now for a discrete function $\mathrm{f}(\mathrm{x})$ the discrete wavelet transform coefficients can be given by :

$$
W_{\psi}\left(j_{0}, i\right)=\frac{1}{\sqrt{m}} \sum_{x} f(x) \psi_{j, i}(x)
$$

where $\psi_{j, i}(x)$ is the sampled version of the scaling function, $\psi_{j, i}(t)$.

$$
\psi_{j, i}(t)=2^{\frac{j}{2}} \psi\left(2^{j} t-i\right)
$$

Single level DWT is the simplest one that decomposes an image into four sub-band images that is LL, LH, HL and $\mathrm{HH}$. Out of these four bands, the LL band is the sub band in which the pixel values of the input image are changing very slowly whereas in rest of the sub bands, the pixel values are changing quickly.

Super resolution in this case can be achieved by interpolating the high frequency sub-bands and the input image as shown in figure 2. While applying IDWT, input image is used instead of LL sub-band image since the input low resolution image contains more information.

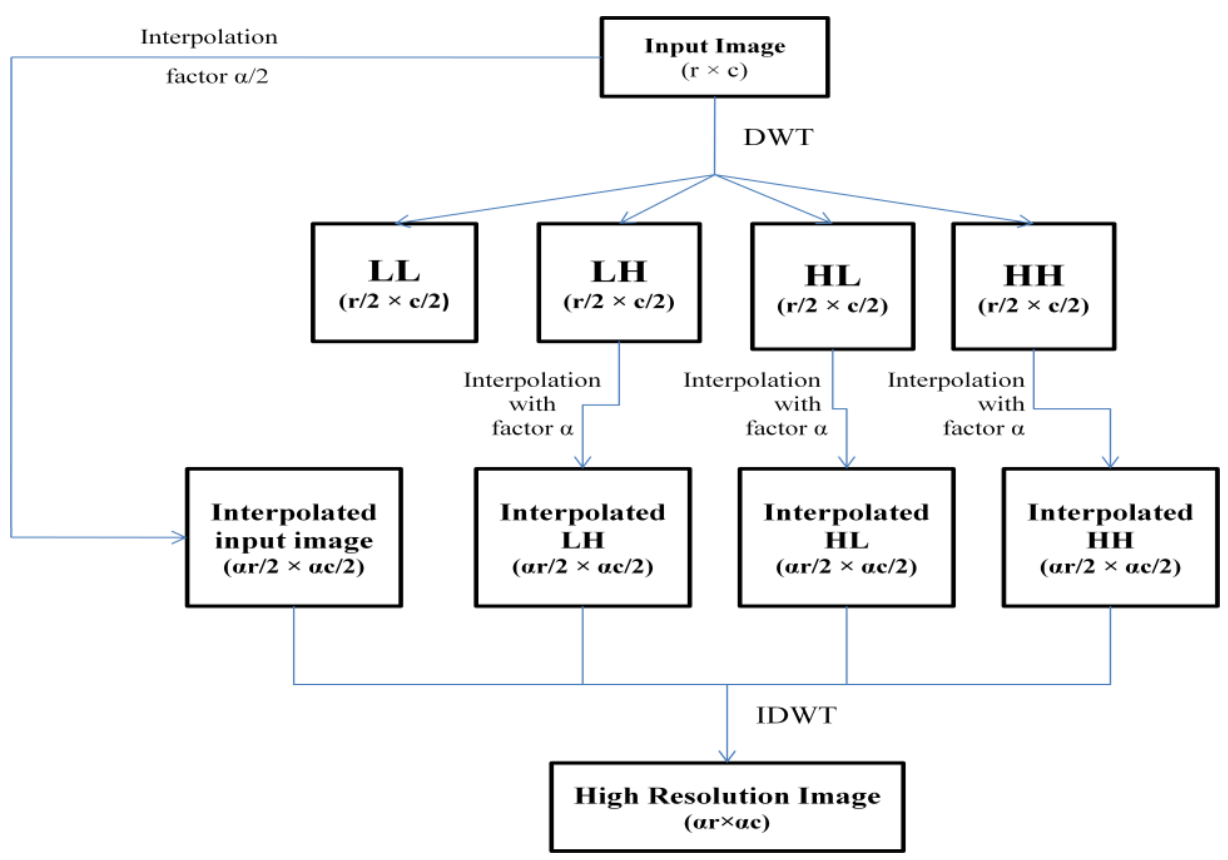

Figure 2: Block diagram of DWT based SR 


\subsection{Stationary wavelet transform}

This wavelet is a bit different from discrete wavelet transform in the fact that it does not down-sample the image sub-bands. Hence the size of the sub-band image is same as that of the original input image. The main purpose of using this wavelet is that it minimizes the information loss and hence enhances the edges. It can be used along with discrete wavelet transform to enhance the low resolution input image. The decomposition analysis using SWT can be visualized in the following figure 3 where it does not use down-sampling.

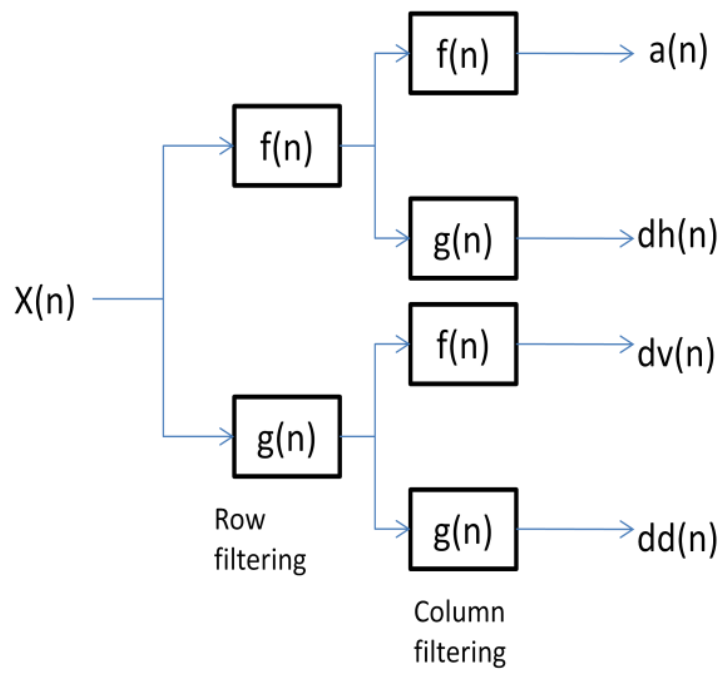

Figure 3: Decomposition analysis of two dimensional SWT

The sub-band images obtained by using SWT are of same size as that of the input image and are added to the interpolated new high frequency sub-bands to give the estimated high frequency bands. Inverse DWT is performed on these estimated high frequency sub-bands and the input image to give the high resolution output image. The low frequency subband (LL) represents the low resolution part of the input image, that is, it contains comparatively lesser amount of information than the input image. Hence the input low resolution image is used instead of the LL sub-band to enhance the quality of the output image.

\section{PROPOSED METHOD}

Interpolation is a technique used to augment the amount of pixels in an image using any of the three interpolation approaches namely (i) nearest neighbour interpolation, (ii) bilinear interpolation, (iii) bi-cubic interpolation.

In this proposed method of obtaining a highly resolved output image from an input image of low resolution, the input image is first decomposed into four sub-band images i.e. LL, LH, $\mathrm{HL}$ and $\mathrm{HH}$ where the low frequency sub-band (LL) contains the low frequency components of the input image and rest of the sub-bands contains the high frequency components. The difference image, $D(i, j)$, is obtained by deducting the interpolated LL sub-band image from the input image. The difference is then appended to the interpolated high frequency sub-bands to give new LH, new HL and new $\mathrm{HH}$.

$$
\begin{aligned}
D(i, j) & =I(i, j)-L L_{i \text { terp }}(i, j) \\
\text { new } L H & =D(i, j)+L H_{\text {interp }}(i, j) \\
\text { new } H L & =D(i, j)+H L_{\text {interp }}(i, j) \\
\text { new } H H & =D(i, j)+H H_{\text {interp }}(i j)
\end{aligned}
$$

where $I(i, j)$ denotes the input image, $L_{\text {interp }}(i, j)$ is the interpolated $\mathrm{LL}$ sub band, $\mathrm{LH}_{\text {interp }}(\mathrm{i}, \mathrm{j})$ is the interpolated $\mathrm{LH}$ sub band and so on.

While using DWT, the sub-bands are down-sampled which causes some information loss. To avoid this information loss, another wavelet that is stationary wavelet transform is employed.

\section{IMAGE QUALITY ANALYSIS}

The quality of the output image can be analyzed using a factor called Peak Signal to Noise Ratio (PSNR) [12]. It tells us how the original image is affected by the introduction of noise. It is basically defined as the ratio of maximum possible power of the signal and noise power. A higher PSNR generally indicates a good quality of reconstructed image and it is similar to the original one. It is measured in decibels. For calculating the value of PSNR, first we need to compute mean squared error which is given by:

$$
M S E=\frac{1}{r c} \sum_{i=0}^{r-1} \sum_{i=0}^{c-1}[I(i, j)-O(i, j)]^{2}
$$

where $I(i, j)$ and $O(i, j)$ corresponds to the original input image and the output reconstructed image respectively. $\mathrm{r}$ and $\mathrm{c}$ are the rows and columns of the image.

PSNR may be given by the following equation.

$$
\begin{aligned}
& \operatorname{PSNR}(d b)=10 * \log _{10}\left(\frac{R^{2}}{M S E}\right) \\
& =20 * \log _{10}(R / \sqrt{M S E}) \\
& =20 * \log _{10}(R)-10 * \log _{10}(M S E)
\end{aligned}
$$

where $\mathrm{R}$ is the maximum possible pixel value of the image. In this case the pixels can be represented using eight (8) bits per samples and hence $\mathrm{R}$ is taken as 255 .

In the absence of noise in the output image $(\mathrm{O})$ then the MSE will be zero and Peak signal to noise ratio will be infinity.

\section{EXPERIMENTAL RESULTS}

The super resolution technique discussed in this paper has been applied to images obtained using CT scan and MRI system. Note that the images obtained using imaging systems have been down-sampled to get the low resolution images. Then the algorithm is applied to get the high resolution output image which is then compared to the original high resolution image.

Figure 4 shows that the high resolution biomedical output image obtained using the proposed method is far better than the output images obtained through other techniques. The proposed method not only increases the clarity of the output image but the size of the output image is also doubled.

This technique as shown works well both qualitatively and quantitatively. 


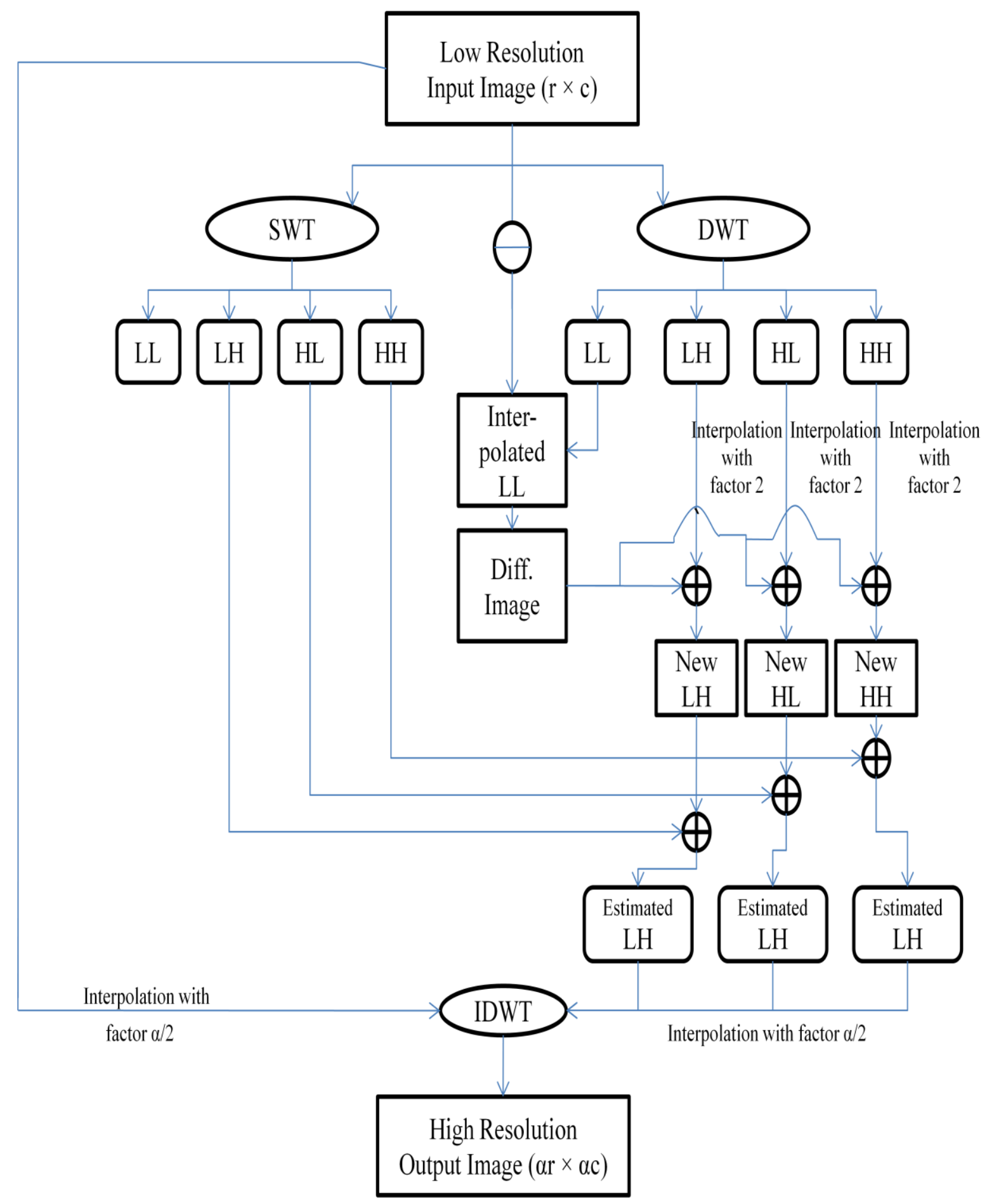

Figure 4: Block diagram of the proposed SR technique 


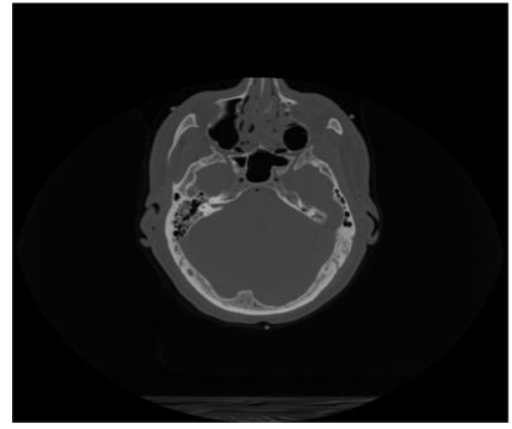

(a)

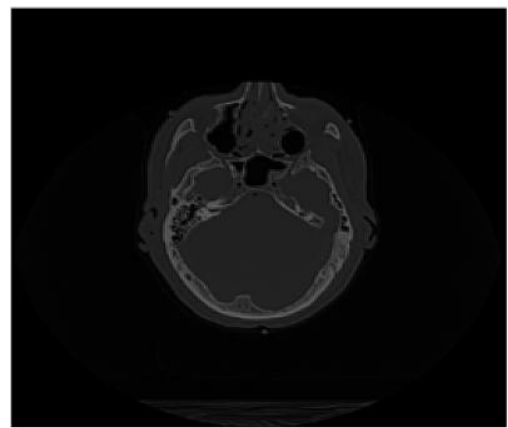

(c)

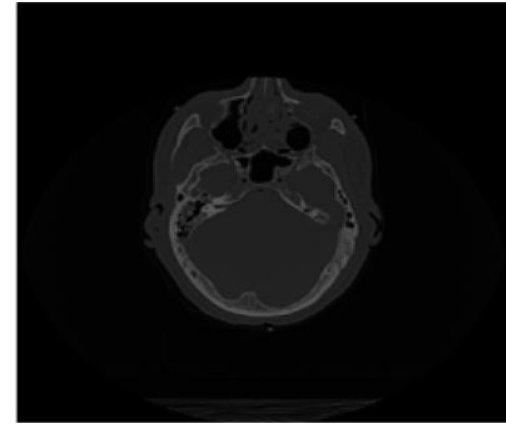

(b)

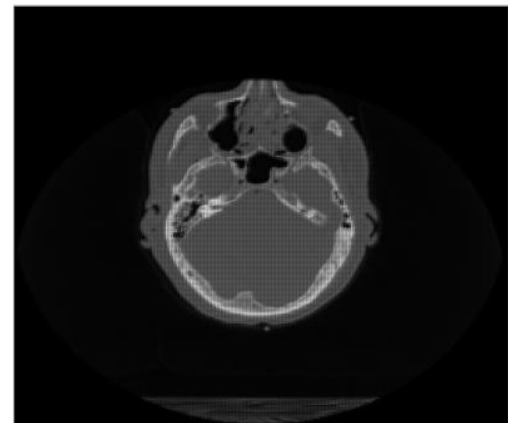

(d)

Figure 5: CT scan of head subset $(512 \times 512)$ (a) Original high resolution (HR) image (b) DWT based high resolution image (c) DWT and SWT based high resolution image (d) Result of the proposed SR method. The proposed method produces high resolution image with sharp edges and high clarity.

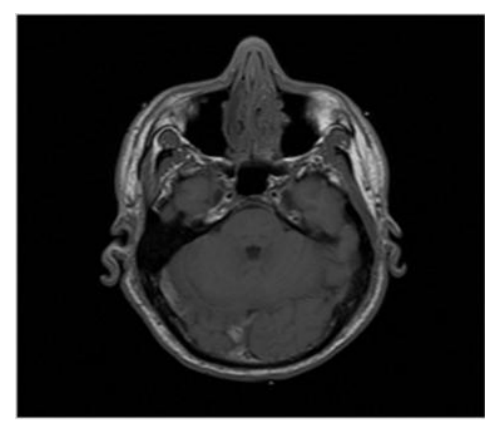

(a)

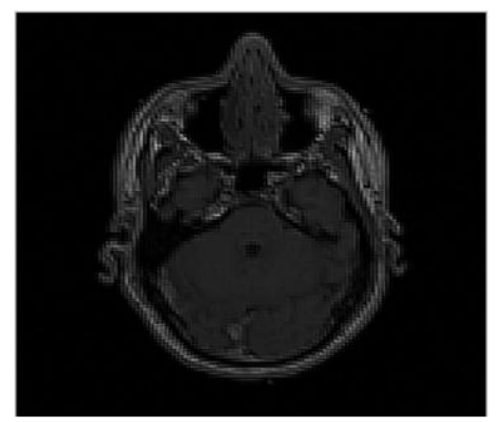

(c)

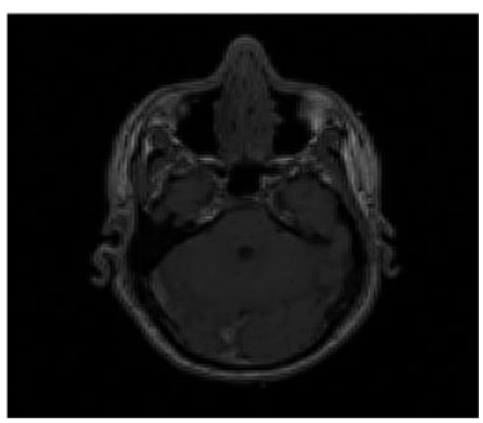

(b)

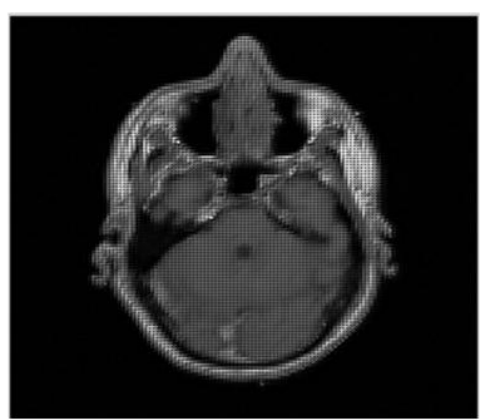

(d)

Figure 6: MRI of head subset $(256 \times 256)$ (a) original high resolution image (b) DWT based high resolution image (c) DWT and SWT based high resolution image (d) Result of the proposed SR technique. 
Table 1: CT scan image results from $256 \times 256$ to $512 \times 512(\alpha=2)$ of the proposed technique

\begin{tabular}{|c|c|c|}
\hline Techniques & PSNR(db) & RMSE \\
\hline Bi-cubic interpolation & 42.26 & 1.96 \\
\hline Bilinear interpolation & 40.21 & 2.48 \\
\hline Discrete wavelet transform & 22.90 & 18.24 \\
\hline SWT and DWT based SR & 23.57 & 18.95 \\
\hline Proposed technique & 65.10 & 0.14 \\
\hline
\end{tabular}

Table 2: MRI results from $128 \times 128$ to $256 \times 256(\alpha=2)$ of the proposed technique

\begin{tabular}{|c|c|c|}
\hline Techniques & PSNR(db) & RMSE \\
\hline Bi-cubic interpolation & 33.25 & 5.545 \\
\hline Bilinear interpolation & 31.83 & 6.528 \\
\hline Discrete wavelet transform & 20.70 & 23.51 \\
\hline SWT and DWT based SR & 20.00 & 25.80 \\
\hline Proposed technique & 63.09 & 0.178 \\
\hline
\end{tabular}

Table 1 and table 2 compare the PSNR values obtained by the proposed method with those obtained by bi-cubic interpolation, bilinear interpolation, DWT based SR, DWT and SWT based SR. It can be seen that the values of PSNR as much as $25 \mathrm{db}$ higher than other methods has been observed. The proposed technique is working well for different image sizes.

\section{CONCLUSIONS}

In this paper we have proposed a new super resolution technique which is based on the interpolation of high frequency sub-band images obtained by discrete wavelet transform and the low resolution input image. Information loss due to down sampling is reduced using SWT. An intermediate stage is introduced and applied to extract more high frequency information. The SR method not only enlarges the low resolution input biomedical image but also restores the degraded image. Improvement in the super resolved image is seen both visually and quantitatively.

\section{REFERENCES}

[1] A.Lapini, F.Argenti, A.Piva, " Comparison of super resolution methods for Quality enhancement of digital biomedical images" in ISMICT , $8^{\text {th }}$ international symposium , April 2014.

[2] K. Zhang, X.Gao, D.Tao, “ Single image Super resolution with multiscale similarity learning", in IEEE transaction, vol 24, Oct 2013.

[3] D. Glasner, S. Bagon, and M. Irani, "Super-resolution from a single image", in proc. IEEE Int. Conf. Comput. Vis., Oct. 2009, pp. 349-356.

[4] Shah, A.J , Gupta, S.B, “ Image super resolution - a survey” in ET2ECN on Dec 2012.
[5] X. Li and M. T. Orchard, "New edge-directed interpolation," IEEE Trans. Image Process., vol. 10, no. 10, pp. 1521-1527, Oct. 2001.

[6] Z. C. Lin and H. Y. Shum," Fundamental limits of reconstruction based super resolution algorithms under local translation," IEEE Trans. Pattern Anal. Mach. Intell., vol. 6, no. 1, pp. 83-97, Jan. 2004.

[7] K. Zhang, X. Gao, D. Tao and X. Li, " Single image super resolution with non-local means and steering kernel regression," IEEE Trans. Image Process., vol. 21, no. 11, pp. 4544-4556, Nov. 2012.

[8] G. Anbarzafari and H. Demirel, "Image super resolution method based on interpolation of wavelet domain high frequency subbands and the spatial domain input image," ETRI J., vol. 32, no. 3, pp. 390-394,july2010.

[9] H. Demirel and G. Anbarjafari, "Satellite image resolution enhancement using complex transform," IEEE Geosci. Remote Sens. Lett., vol. 7, no. 1, pp. 123 126, Jan. 2010.

[10] G. Anbarzafari and H. Demirel," Image resolution enhancement by using discrete and stationary wavelet decomposition", IEEE transactions on image processing, vol. 20, no. 5, May 2011

[11] M. Vinay Kumar, Sri. P. Madhukiran,"Multi resolution analysis based MRI image quality analysis using DTCWT based preprocessing techniques", International Journal of Engineering Research and General Science, vol. 2, Issue 5, August-September 2014.

[12] Hore. A, Ziou. D, "Image Quality Metrics: PSNR vs. SSIM", in 2010 International conference on pattern recognition, pp-2366-2369, 23-26 August 2010. 